\title{
The Internal Structure of White Dwarf Stars Using the Whole Earth Telescope
}

\author{
P.A. Bradley \\ McDonald Observatory and Department of Astronomy, University of Texas
}

\begin{abstract}
White dwarfs are the final end state for the majority of stars, and hold clues to help solve many current pressing astrophysical problems. We can perform asteroseismology on the pulsating white dwarfs to better understand their internal structure and input physics, paving the way to a better understanding of astrophysics, stellar evolution, and the history of our Galaxy. I describe briefly the potential of asteroseismology by using it to infer the internal structure of PG1159-035.
\end{abstract}

\section{What Are White Dwarfs and Why Are They Interesting?}

White dwarfs have attracted considerable interest from astronomers because they offer insight into many problems of physics and astrophysics. In the following discussion, I will concentrate on the pre-white dwarfs known as PG1159 stars. Like the white dwarfs they will become, the PG1159 stars are the product of stellar evolution with main sequence masses up to $\sim 8 \mathrm{M} \odot$, and have an average mass of $0.5-0.6 \mathrm{M} \odot$ (see Bergeron, Saffer, \& Liebert 1992; Weidemann 1990). We believe the PG1159 stars have carbon/oxygen cores comprising more than $99 \%$ of the mass, and spectroscopy (Werner, Heber, \& Hunger 1991) reveals ionized carbon, helium, oxygen, and sometimes nitrogen in an atmosphere with a temperature of 100,000 to $150,000 \mathrm{~K}$. The PG1159 stars will eventually become either helium atmosphere (DB) white dwarfs or hydrogen atmosphere (DA) white dwarfs. Nature is kind enough to supply us with pulsating PG1159 stars, DB and DA white dwarfs for seismology so that can determine the evolutionary fate of the PG1159 stars. (See the conference proceedings by Vauclair \& Sion 1991; Wegner 1989; and Philip, Hayes, \& Liebert 1987 for articles concerning the structure, evolution, and pulsations of white dwarfs.)

The intense gravitational fields $(\log g \sim 7-8)$ of the PG1159 stars and white dwarfs implies that they should have a layered structure, with the heavier elements (carbon + oxygen) sinking to become the core, while the lighter helium and hydrogen float to the surface. The compositional stratification expected of white dwarf stars means that a $g$-mode can become trapped within a given region of the star when the wavelength of the $g$-mode matches the thickness of the trapping layer, allowing a resonance to form. (See Brassard et al. 1992 and references therein for more details on mode trapping in white dwarfs.) Observationally, mode trapping reveals itself 
through minima in the period spacing between consecutive overtone modes, the result of modes in or near the mode trapping resonance having very similar eigenfunctions and pulsation periods. Models with thicker surface layers require lower overtone modes for the first mode trapping resonance to form, and the number of nontrapped modes in between the trapped modes is smaller. Thus, if the mass of the surface layer is close to that predicted by stellar evolution calculations (see Iben 1991; Mazzitelli \& D'Antona 1990; and references therein) the interval between period spacing minima will be shorter than for the much thinner surface layer masses predicted by spectral evolution theory (Fontaine \& Wesemael 1991, 1987).

\section{Sample Results}

New observations from the Whole Earth Telescope (E WET; Nather et al. 1990) have resolved the light curves of several pre-white dwarf and white dwarf stars with unprecedented detail. The analysis of WET data on the pre-white dwarf PG1159035 (Winget et al. 1991) demonstrates that asteroseismology can yield much new information about these stars with existing asymptotic theory and models. However, these WET observations, along with those of other white dwarfs, demonstrate that we need a new generation of models for asteroseismological determinations of their structure.

As an example of the potential of the new models (Wood 1990), we show plots of the period spacing between consecutive overtone modes versus the pulsation period for PG1159-035 (a DOV star) against the predicted periods from some of the new models. For PG1159-035, the interval between period spacing minima-trapped modes-is best matched by models with a transition region at a few $\times 10^{-3} M_{\star}$ (see Figure 1). At long periods, the period spacing curve for the model is much flatter than the observations, suggesting that we don't yet have the composition transition region accurately modelled. We can add this to what we already know about PG1159-035 from the previous efforts of Winget et al. (1991), who determined the total stellar mass $(0.586 \mathrm{M} \odot)$, the rotation rate $(1.38 \mathrm{~d})$, set limits on any magnetic field that may be present $(<6000 \mathrm{G})$, and demonstrated the star must already be stratified. More work on the model matching should allow us to arrive at a better match to the observations and a better knowledge of PG1159-035's internal structure, although we can already say that the surface layer mass is several times less than the value predicted by stellar evolution theory.

Clearly, the prospect of asteroseismology for stellar structure determination is quite promising. With time, we expect to resolve and interpret the observed period structure of many pulsating white dwarfs to better understand their structure and place in the universe.

I am grateful to C.J. Hansen, S.D. Kawaler, R.E. Nather, D.E. Winget, and M.A. Wood for their help, encouragement, and many discussions. This research was supported by the National Science Foundation under grants 85-52457 and 90-14655 through the University of Texas and McDonald Observatory. 


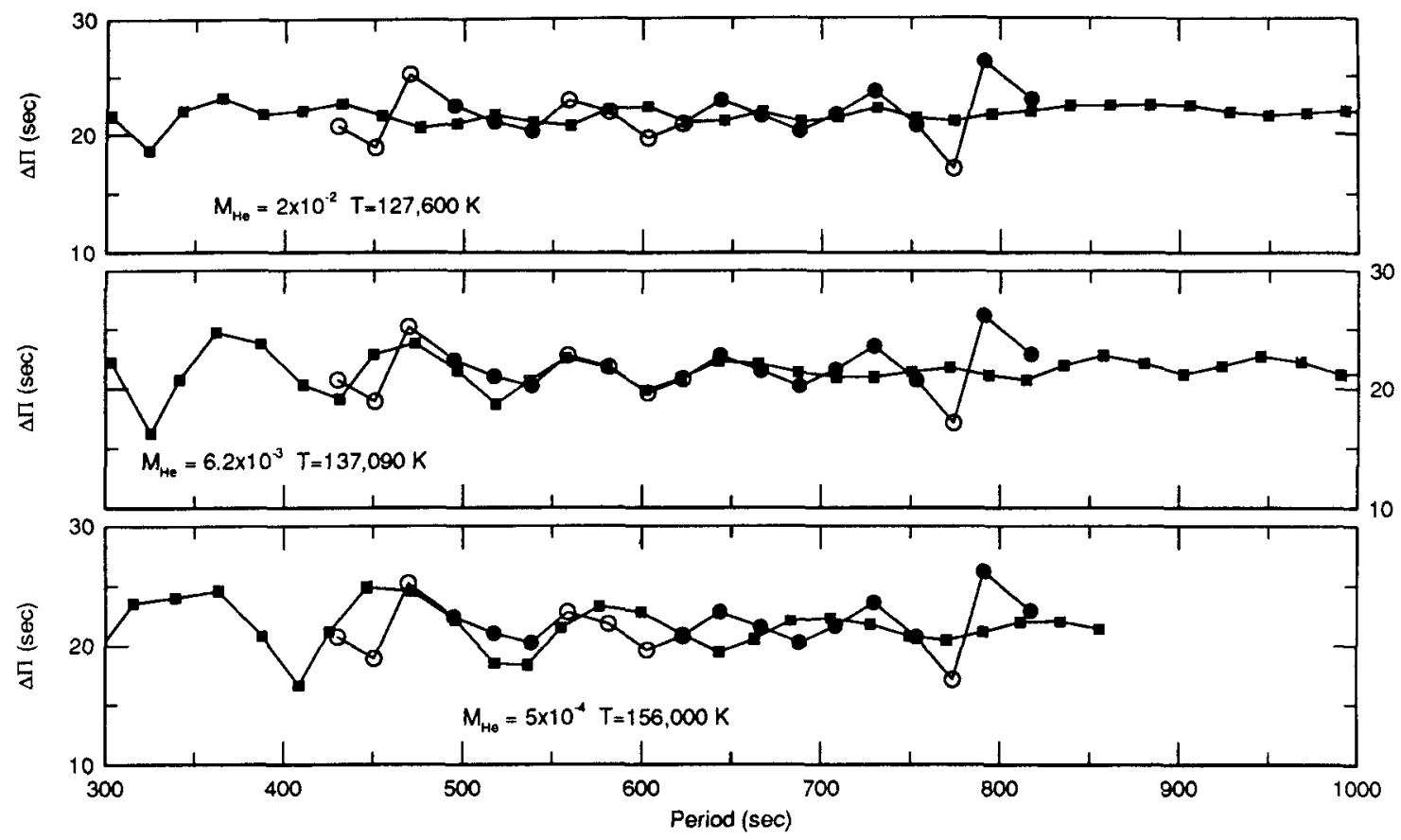

Figure 1: Sample fits of the $\ell=1$ period spacings for PG1159-035 (dots) versus period spacings from $0.59 \mathrm{M} \odot$ evolutionary models (squares).

The open dots represent less certain period determinations than the solid dots.

\section{References:}

Bergeron, P., Saffer, R.A., \& Liebert, J. 1992, ApJ, 394, 228

Brassard, P., Fontaine, G., Wesemael, F. \& Hansen, C.J. 1992, ApJS, 80, 369

D'Antona, F., \& Mazzitelli, I., 1990, ARA\&A, 28, 139

Fontaine, G., \& Wesemael, F. 1991, in IAU Symp. 145, The Photospheric Abundance Connection, ed. G. Michaud \& A. Tutukov, (Dordrecht: Reidel), 421

Iben, I.,Jr. 1991, ApJS, 76, 55

Nather, R.E., Winget, D.E., Clemens, J.C., Hansen, C.J., \& Hine, B.P. 1990, ApJ, 361, 309

Philip, A.G.D., Hayes, D.S., \& Liebert, J. 1987, IAU Colloq. 95, The Second Conference on Faint Blue Stars, (Schenectady: Davis)

Starrfield, S.G., Cox, A.N., Kidman, R.B., \& Pesnell, W.D. 1984, ApJ, 281, 800

Vauclair, G., \& Sion, E.M. 1991, in the Seventh European Workshop on White Dwarfs, White Dwarfs, (NATO ASI Ser.), (Dordrecht: Kluwer)

Wegner, G. 1989, IAU Colloq. 114, White Dwarfs, (Berlin: Springer)

Werner, K., Heber, U., \& Hunger, K. 1991, A\&A, 244, 437

Weidemann, V. 1990, ARA\&A, 28, 103

Winget, D.E. 1988, in IAU Symposium 123, Advances in Helio- and Asteroseismology, ed.

J. Christensen-Dalsgaard \& S. Frandsen, (Dordrecht: Reidel), 305

Winget, D.E., et al. 1991, ApJ, 378, 326

Wood, M.A. 1990, Ph.D. thesis, University of Texas at Austin 